\title{
Anisotropy of Cubic Ferromagnet at Criticality
}

\author{
A. Kudlis ${ }^{1,2}$ and A. I. Sokolov ${ }^{1 *}$ \\ ${ }^{1}$ Division of Quantum Mechanics, Saint Petersburg State University, \\ Ulyanovskaya 1, Petergof, Saint Petersburg, 198504 Russia, \\ ${ }^{2}$ ITMO University, Kronverkskii ave 49, Saint Petersburg 197101, Russia
}

(Dated: April 4, 2018)

\begin{abstract}
Critical fluctuations change the effective anisotropy of cubic ferromagnet near the Curie point. If the crystal undergoes phase transition into orthorhombic phase and the initial anisotropy is not too strong, reduced anisotropy of nonlinear susceptibility acquires at $T_{c}$ the universal value $\delta_{4}^{*}=\frac{2 v^{*}}{3\left(u^{*}+v^{*}\right)}$ where $u^{*}$ and $v^{*}$ - coordinates of the cubic fixed point on the flow diagram of renormalization group equations. In the paper, the critical value of the reduced anisotropy is estimated within the pseudo- $\epsilon$ expansion approach. The six-loop pseudo- $\epsilon$ expansions for $u^{*}, v^{*}$, and $\delta_{4}^{*}$ are derived for the arbitrary spin dimensionality $n$. For cubic crystals $(n=3)$ higher-order coefficients of the pseudo- $\epsilon$ expansions obtained turn out to be so small that use of simple Padé approximants yields reliable numerical results. Padé resummation of the pseudo- $\epsilon$ series for $u^{*}, v^{*}$, and $\delta_{4}^{*}$ leads to the estimate $\delta_{4}^{*}=0.079 \pm 0.006$ indicating that detection of the anisotropic critical behavior of cubic ferromagnets in physical and computer experiments is certainly possible.

PACS numbers: 05.10.Cc, 05.70.Jk, 64.60.ae
\end{abstract}




\section{INTRODUCTION}

Thermal fluctuations of the order parameter are known to change effective anisotropy of a cubic ferromagnet approaching the Curie point. As was first discovered by Wilson and Fisher a system with cubic symmetry may become isotropic under $T \rightarrow T_{c}$ provided its initial anisotropy is small enough 1 . On the contrary, if the "bare" anisotropy is big it increases further until the fluctuation-driven discontinuous phase transition occurs ${ }^{2}-\underline{\underline{4}}$. If a ferromagnet undergoes the second-order phase transition, i. e. its initial anisotropy is not too strong, and the low-temperature phase is orthorhombic, under $T \rightarrow T_{c}$ the anisotropy of magnetic subsystem acquires the universal value which does not depend on its magnitude far from the Curie point. As was revealed in the course of the study of critical behavior of $n$-vector cubic model, the order parameter dimensionality $n$ plays a key role here: for $n<n_{c}$ the system undergoing continuous phase transition demonstrates isotropic critical behavior while for $n>n_{c}$ it remains anisotropic at $T_{c}$.

The numerical value of the marginal spin dimensionality $n_{c}$ separating these two regimes is of prime physical importance since it determines the true mode of the critical behavior of real cubic ferromagnets. Early estimates of $n_{c}$ deduced from the lower-order renormalizationgroup $(\mathrm{RG})$ calculations ${ }^{3,6.7}$ turned out to be in favor of the inequality $n_{c}>3$ implying that cubic ferromagnets should belong to the class of universality of the three-dimensional (3D) Heisenberg model. The results obtained within the high-temperature expansion approach seemed to support this conclusion- ${ }^{8}$. However, the resummation of the three-loop RG expansions $\frac{9}{10}$ and subsequent higher-order RG analysis both in three $\underline{11}-\underline{13}$ and $(4-\epsilon) \underline{14}$ dimensions as well as advanced lattice calculations shifted the value of $n_{c}$ downwards fixing it below $3^{9-22}$. Impressive consensus between different field-theoretical approaches and resummation techniques was achieved in the course of this study: 3D RG calculations $\frac{13}{}$, resummed $\epsilon$ expansions $\frac{17}{17}$, biased $\epsilon$ expansion technique ${ }^{13}$, and pseudo- $\epsilon$ expansion analysis $\frac{19}{}$ yielded $n_{c}=2.89, n_{c}=2.855, n_{c}=2.87$, and $n_{c}=2.862$, respectively. Thus, cubic ferromagnets undergoing second-order phase transitions should demonstrate the anisotropic critical behavior with a specific set of critical exponents.

On the other hand, since $n_{c}$ is very close to the physical value $n=3$, the cubic fixed point lies very near the Heisenberg fixed point at the RG flow diagram. It implies that the critical exponents for both fixed points should almost coincide. Indeed, the susceptibility exponent 
$\gamma$, for instance, is equal to $1.3895(50)$ for the Heisenberg fixed point ${ }^{23}$ and to $1.390(6)$ for the cubic one $\underline{13}$. As a result, measuring critical exponents in physical or computer experiments one can not distinguish between the cubic and Heisenberg critical behaviors.

To clear up how the system with cubic symmetry behaves near $T_{c}$ one should explore some alternative physical quantities. It was suggested $\underline{24}$ that nonlinear susceptibilities of different orders can play a role of indicators of anisotropic critical behavior. The RG analysis of the lowest-order nonlinear susceptibility $\chi^{(4)}$, in particular, has shown that its reduced cubic anisotropy may be as large as $5 \%$ at criticality 24, i. e. is certainly detectable experimentally. At the same time, although this estimate was extracted from the longest - six-loop - RG expansions available it is hardly believed to be quite reliable. The point is that the anisotropy of $\chi^{(4)}$ is evaluated via the cubic fixed point coordinates $u_{4}^{*}$ and $v_{4}^{*}$ and one of them $\left(v_{4}^{*}\right)$ being numerically small can not be found with high accuracy on the base of the diverging series for $\beta$ functions.

In such a situation it looks natural to analyze the nonlinear susceptibility of cubic ferromagnets and its anisotropy near $T_{c}$ using alternative approaches. In this paper, the anisotropy of $\chi^{(4)}$ of the 3D cubic model will be studied in the framework of the pseudo- $\epsilon$ expansion technique. This approach invented by B. Nickel (see Ref. 19 in the paper of Le Guillou and Zinn-Justin吕) exploits the idea that the fixed point location in three dimensions may be found iteratively by means of introducing fictitious small parameter $\tau$ into linear terms of the perturbative series for $\beta$ functions. The method of pseudo- $\epsilon$ expansion proved to be very efficient when used to estimate critical exponents and other universal quantities of various three-dimensional systems $\underline{19}, 23,25-36$. Moreover, even in two dimensions where RG series are shorter and more strongly divergent it enables one to get fair numerical results $257-41$. The numerical power of the pseudo- $\epsilon$ expansion machinery, i. e. its ability to accelerate RG iterations and to smooth oscillations of numerical estimates as functions of the order of approximation, is so high that in many cases pseudo- $\epsilon$ expansions ( $\tau$-series) employed do not require advanced resummation procedures. As a rule, use of Padé approximants or even direct summation turns out to be sufficient to lead to proper numerical results.

The paper is organized as follows. In Section 2 the nonlinear susceptibility $\chi^{(4)}$ of the cubic model is discussed, the parameter $\delta_{4}$ characterizing its anisotropy is introduced, and the relation between $\delta_{4}$ and effective quartic coupling constants $u_{4}, v_{4}$ is presented. In 
Section 3 pseudo- $\epsilon$ expansions for the cubic fixed point coordinates $u_{4}^{*}$ and $v_{4}^{*}$ and for the critical value of the reduced anisotropy $\delta_{4}$ are derived for arbitrary $n$. The $\tau$-series for $u_{4}^{*}, v_{4}^{*}$, and $\delta_{4}^{*}$ at $n=3$ which are of particular physical interest are presented in Section 4 . Here the resummation of the pseudo- $\epsilon$ expansions obtained is carried out and the numerical estimate for the reduced anisotropy at criticality is presented. In Section 5 this estimate is compared with those given by the 3D RG analysis, their deviation from each other is discussed, and the robustness of the pseudo- $\epsilon$ expansion estimate is demonstrated. Conclusion contains the summary of the main results obtained.

\section{QUARTIC COUPLING CONSTANTS, NONLINEAR SUSCEPTIBILITY, AND ITS ANISOTROPY}

Near Curie point the free energy of a cubic ferromagnet may be written down as a series in powers of magnetization components $M_{\alpha}$ :

$$
F\left(M_{\alpha}, m\right)=F(0, m)+\frac{1}{2} m^{2-\eta} M_{\alpha}^{2}+m^{1-2 \eta}\left(u_{4}+v_{4} \delta_{\alpha \beta}\right) M_{\alpha}^{2} M_{\beta}^{2}+\ldots
$$

where $\eta$ is a Fisher exponent, $m$ being an inverse correlation length while $u_{4}$ and $v_{4}$ are dimensionless effective coupling constants that acquire under $T \rightarrow T_{c}$ the universal values. These coupling constants are related to the fourth-order nonlinear susceptibility defined in a conventional way:

$$
\chi_{\alpha \beta \gamma \delta}^{(4)}=\left.\frac{\partial^{3} M_{\alpha}}{\partial H_{\beta} \partial H_{\gamma} \partial H_{\delta}}\right|_{H=0} .
$$

Of particular importance are the values of nonlinear susceptibility in two cases, namely when i) an external field is parallel to a cubic axis $\left(\chi_{c}^{(4)}\right)$ and ii) it is oriented along a unit cell space diagonal $\left(\chi_{d}^{(4)}\right)$. For these two directions the difference between corresponding values of nonlinear susceptibility turns out to be maximal, i. e. the anisotropy is most pronounced. As may be readily shown

$$
\chi_{c}^{(4)}=-24 \frac{\chi^{2}}{m^{3}}\left(u_{4}+v_{4}\right), \quad \chi_{d}^{(4)}=-24 \frac{\chi^{2}}{m^{3}}\left(u_{4}+\frac{v_{4}}{3}\right),
$$

where $\chi$ is a linear susceptibility. To characterize the anisotropy strength, we define a reduced parameter

$$
\delta_{4}=\frac{\chi_{c}^{(4)}-\chi_{d}^{(4)}}{\chi_{c}^{(4)}}
$$


that is to be estimated at criticality.

Before starting our pseudo- $\epsilon$ expansion calculations let us remind the estimate for the critical value of $\delta_{4}$ originating from the 3D RG analysis. Coordinates of the cubic fixed point $u_{4}^{*}$ and $v_{4}^{*}$ in three dimensions are known today from the higher-order RG calculations, with resummed four-, five-, and six-loop RG expansions yielding close numerical results 11,13 . Considering the six-loop RG estimates as the most reliable ones, we accept $u_{4}^{*}=0.755 \pm 0.010$, $v_{4}^{*}=0.067 \pm 0.014^{13}$. Substitution of these numbers into Eqs. (3), (4) gives

$$
\delta_{4}^{*}=0.054 \pm 0.012
$$

This value will be compared with those resulting from the pseudo- $\epsilon$ expansions for universal values of the quartic couplings as well as from the $\tau$-series for $\delta_{4}^{*}$ itself. These series are derived in the next section.

\section{PSEUDO- $\epsilon$ EXPANSIONS FOR QUARTIC COUPLINGS AND REDUCED ANISOTROPY}

Let us take as a starting point well-known Landau-Wilson Hamiltonian of the 3D $n$-vector cubic model:

$$
H=\frac{1}{2} \int d^{3} x\left[m_{0}^{2} \varphi_{\alpha}^{2}+\left(\nabla \varphi_{\alpha}\right)^{2}+\frac{u_{0}}{12} \varphi_{\alpha}^{2} \varphi_{\beta}^{2}+\frac{v_{0}}{12} \varphi_{\alpha}^{4}\right],
$$

where $m_{o}^{2}$ is proportional to the deviation from the mean-field transition temperature. Our analysis is based on the $\beta$ functions (Gell-Mann-Low functions) of this model calculated within a massive theory under zero-momenta normalizing conditions for the renormalized Green function $G_{R}(p, m)$ and the four-point vertices $U_{R}\left(\mathbf{p}_{\mathbf{1}}, \mathbf{p}_{\mathbf{2}}, \mathbf{p}_{\mathbf{3}}, m, u, v\right)$, $V_{R}\left(\mathbf{p}_{\mathbf{1}}, \mathbf{p}_{\mathbf{2}}, \mathbf{p}_{\mathbf{3}}, m, u, v\right)$ :

$$
\begin{aligned}
G_{R}^{-1}(0, m) & =m^{2}, \quad & \left.\frac{\partial G_{R}^{-1}(p, m)}{\partial p^{2}}\right|_{p^{2}=0} & =1, \\
U_{R}(0,0,0, m, u, v) & =m u, \quad & V_{R}(0,0,0, m, u, v) & =m v .
\end{aligned}
$$

The value of the one-loop graph including the factor $(n+8)$ is absorbed in $u$ and $v$ in order to make the coefficient for $u^{2}$ term in the $\beta_{u}$ function equal to unity. Quartic effective couplings $u$ and $v$ thus defined are related to $u_{4}$ and $v_{4}$ entering Eqs. (1), (3) in a following manner:

$$
u=\frac{n+8}{2 \pi} u_{4}, \quad v=\frac{n+8}{2 \pi} v_{4}
$$


The perturbative expansions for $\beta$ functions of the model (6) are known today up to the six-loop terms $\frac{13}{}$. Although the cubic fixed point of the RG equations is stable only for $n>n_{c}$ we derive the pseudo- $\epsilon$ expansions for coordinates of this point $u^{*}, v^{*}$ under arbitrary $n$. To find them the linear terms in $\beta$ functions are replaced with $\tau u$ and $\tau v$, respectively, where $\tau$ is a fictitious small parameter, and then the equations

$$
\beta_{u}(u, v)=0, \quad \beta_{v}(u, v)=0
$$

are solved iteratively in $\tau$. This procedure results in the pseudo- $\epsilon$ expansions ( $\tau$-series) for $u^{*}$ and $v^{*}$ :

$$
\begin{aligned}
u^{*}= & \frac{(n+8) \tau}{n}\left[\frac{1}{3}-\frac{4(n-1)(85 n-478)}{2187} \frac{\tau}{n^{2}}+(4.5859515-8.6792769 n\right. \\
& \left.+5.4652167 n^{2}-1.1411824 n^{3}+0.0045537001 n^{4}\right) \frac{\tau^{2}}{n^{4}} \\
& +\left(-30.06975+78.40189 n-79.05230 n^{2}+37.61208 n^{3}\right. \\
& \left.\quad-8.073476 n^{4}+0.6043141 n^{5}-0.02075372 n^{6}\right) \frac{\tau^{3}}{n^{6}} \\
& +\left(220.8250-733.6003 n+1007.970 n^{2}-732.1351 n^{3}+298.0330 n^{4}\right. \\
& \left.\quad-66.24082 n^{5}+7.381100 n^{6}-0.4608437 n^{7}+0.02278282 n^{8}\right) \frac{\tau^{4}}{n^{8}} \\
& +\left(-1737.52+7014.10 n-12174.6 n^{2}+11821.6 n^{3}-7004.25 n^{4}+2595.17 n^{5}\right. \\
& \left.\left.\quad-596.900 n^{6}+84.4687 n^{7}-7.88176 n^{8}+0.605252 n^{9}-0.0302510 n^{10}\right) \frac{\tau^{5}}{n^{10}}\right], \quad(10) \\
v^{*}= & \frac{(n+8) \tau}{n}\left[\frac{n-4}{9}+\frac{4(n-1)\left(77 n^{2}+494 n-1912\right)}{6561} \frac{\tau}{n^{2}}+(-6.1146020\right. \\
+ & \left.12.064863 n-7.8052925 n^{2}+1.5927396 n^{3}+0.026369322 n^{4}+0.00065967848 n^{5}\right) \frac{\tau^{2}}{n^{4}} \\
+ & \left(40.09300-107.7651 n+111.0051 n^{2}-52.82124 n^{3}+10.49491 n^{4}\right. \\
- & \left.0.3739251 n^{5}-0.03604614 n^{6}+0.001331510 n^{7}\right) \frac{\tau^{3}}{n^{6}} \\
+ & \left(-294.4333+1001.849 n-1401.721 n^{2}+1025.114 n^{3}-408.9223 n^{4}+82.35319 n^{5}\right. \\
- & \left.5.903868 n^{6}-0.1481744 n^{7}+0.02213800 n^{8}-0.004581224 n^{9}\right) \frac{\tau^{4}}{n^{8}} \\
+ & \left(2316.69-9538.73 n+16819.2 n^{2}-16475.7 n^{3}+9715.12 n^{4}-3480.03 n^{5}+722.129 n^{6}\right. \\
- & \left.\left.76.2769 n^{7}+2.81068 n^{8}+0.105962 n^{9}-0.0352014 n^{10}+0.00445940 n^{11}\right) \frac{\tau^{5}}{n^{10}}\right] .
\end{aligned}
$$

These pseudo- $\epsilon$ expansions should obey some exact relations appropriate to the systems with cubic anisotropy. For $n=2$ the cubic model is known to possess a specific symmetry: 
if the field $\varphi_{\alpha}$ undergoes the transformation

$$
\varphi_{1} \rightarrow \frac{\varphi_{1}+\varphi_{2}}{\sqrt{2}}, \quad \varphi_{2} \rightarrow \frac{\varphi_{1}-\varphi_{2}}{\sqrt{2}}
$$

quartic coupling constants are also transformed:

$$
u \rightarrow u+\frac{3}{2} v, \quad v \rightarrow-v
$$

but the structure of the Hamiltonian itself remains unchanged $\underline{1}$. Since the RG functions of the problem are completely determined by the structure of the Hamiltonian, the RG equations should be invariant with respect to any transformation conserving this structure ${ }^{42}$. This should be also true for all the expressions relating various physical quantities to each other. If, for example, one applies under $n=2$ the transformation (12) to the magnetization components in Eq. (1), the free-energy expansion remains the same, provided $u_{4}, v_{4}$ are replaced according to Eq. (13).

Since the transformation (12), (13) does not influence the structure of the RG equations it should map corresponding flow diagram into itself. This implies, in particular, that the locations of different fixed points at the $(u, v)$ plane should be related to each other. Indeed, if we apply the transformation (13) to the Ising fixed point $\left(0, g_{I}^{*}\right)$ lying on the $v$ axis it will turn into the fixed point with coordinates $\left(3 g_{I}^{*} / 2,-g_{I}^{*}\right)$, i. e. into the cubic fixed point, and vice versa. Both fixed points have similar character of stability - they are unstable (saddle) fixed point. This similarity is quite natural since because of the specific symmetry mentioned these points are equivalent being, in fact, the same fixed point.

For $n=2$ the pseudo- $\epsilon$ expansions for the coordinates of the cubic fixed point resulting from (10), (11) are as follows

$$
\begin{gathered}
u^{*}=\frac{5}{3} \tau+\frac{1540}{2187} \tau^{2}+0.0098952 \tau^{3}+0.019972 \tau^{4}-0.06872 \tau^{5}+0.0669 \tau^{6}, \\
v^{*}=-\frac{10}{9} \tau-\frac{3080}{6561} \tau^{2}-0.0065968 \tau^{3}-0.013315 \tau^{4}+0.04581 \tau^{5}-0.0446 \tau^{6} .
\end{gathered}
$$

Under the transformation (13) the cubic fixed point turns into the Ising one. Hence, the sum $u^{*}+3 v^{*} / 2$ should be equal to zero. As is seen, $\tau$-series (14) and (15) do really obey the relation $u^{*}+3 v^{*} / 2=0$. Moreover, due to the same symmetry the series (15) should coincide with the pseudo- $\epsilon$ expansion for the Wilson fixed point coordinate $g_{I}^{*}$ of the 3D Ising model. 
One can easily check, making obvious rescaling, that the expansion (15) is identical to the six-loop $\tau$-series for $g_{I}^{*}$ reported in Ref. 26 .

At the end of this section we present the pseudo- $\epsilon$ expansion for the reduced anisotropy $\delta_{4}^{*}:$

$$
\begin{aligned}
\delta_{4}^{*} & =\frac{2(n-4)}{3(n-1)}+\frac{16(n-2)}{9 n(n-1)} \tau+\frac{(n-2)}{n^{3}(n-1)}\left(-4.6627082+5.0533359 n-0.76655300 n^{2}\right) \tau^{2} \\
& +\frac{(n-2)}{n^{5}(n-1)}\left(24.45841-43.98925 n+28.53713 n^{2}-5.962430 n^{3}+0.4618007 n^{4}\right) \tau^{3} \\
& +\frac{(n-2)}{n^{7}(n-1)}\left(-160.375+403.068 n-408.619 n^{2}+207.490 n^{3}-52.3182 n^{4}+6.04176 n^{5}\right. \\
& \left.-0.431021 n^{6}\right) \tau^{4}+\frac{(n-2)}{n^{9}(n-1)}\left(1177.7-3801.7 n+5206.8 n^{2}-3920.4 n^{3}+1754.3 n^{4}\right. \\
& \left.-469.54 n^{5}+76.035 n^{6}-7.3777 n^{7}+0.52362 n^{8}\right) \tau^{5} .
\end{aligned}
$$

Note that all the terms of the series (16) apart from the first one vanish when $n \rightarrow 2$. This reflects the specific symmetry discussed above which enables, in particular, to find the exact value of the reduced anisotropy under $n=2$. Indeed, substituting the coordinates of the cubic fixed point $\left(3 g_{I}^{*} / 2,-g_{I}^{*}\right)$ into the relation

$$
\delta_{4}^{*}=\frac{2 v^{*}}{3\left(u^{*}+v^{*}\right)}
$$

resulting from (3) and (4) we obtain $\delta_{4}^{*}=-4 / 3$ for $n=2$. The pseudo- $\epsilon$ expansion (16) is seen to be in accord with this result.

\section{NUMERICAL ESTIMATES FOR CUBIC FERROMAGNETS $(n=3)$}

Let us use the pseudo- $\epsilon$ expansions just obtained to estimate the critical values of effective coupling constants and reduced anisotropy for cubic ferromagnets, i. e. in the physical case $n=3$. Relevant $\tau$-series are as follows:

$$
\begin{gathered}
u^{*}=\frac{11}{9} \tau+\frac{19624}{59049} \tau^{2}-0.12258455 \tau^{3}-0.0655945 \tau^{4}-0.061083 \tau^{5}+0.01269 \tau^{6}, \\
v^{*}=-\frac{11}{27} \tau+\frac{23144}{177147} \tau^{2}+0.23233729 \tau^{3}+0.1283989 \tau^{4}+0.050252 \tau^{5}+0.02224 \tau^{6}, \\
\delta_{4}^{*}=-\frac{1}{3}+\frac{8}{27} \tau+0.06663560 \tau^{2}+0.0529734 \tau^{3}-0.025228 \tau^{4}+0.03848 \tau^{5} .
\end{gathered}
$$


These expansions originating from the diverging $\mathrm{RG}$ series for $\beta$ functions are divergent as well. Hence, to obtain from (18)-(20) the numbers of interest proper resummation procedures should be applied. On the other hand, since these series have small higher-order coefficients a direct summation may result in fair numerical estimates. When applied to the series (20) it yields, under $\tau=1, \delta_{4}^{*}=0.096$. This number differs considerably from the estimate $\delta_{4}^{*}=0.054$ given by the $3 \mathrm{D}$ RG analysis $\underline{13}, \underline{24}$. The estimate just found may be corrected if we accept that the pseudo- $\epsilon$ expansions in hand are asymptotic and one can get the best numerical results cutting off the series by smallest terms. Such an optimally truncated direct summation of $\tau$-series (20) leads to $\delta_{4}^{*}=0.057$. An alternative way to estimate $\delta_{4}^{*}$ with a help of direct summation is to find the universal values of quartic coupling constants and to use the relation (17). Direct summation of the series (18), (19) under $\tau=1$ gives $u^{*}=1.318, v^{*}=0.156$ resulting in $\delta_{4}^{*}=0.071$.

The estimates of $\delta_{4}^{*}$ thus obtained are appreciably scattered and obviously need to be refined. Since the coefficients of all the $\tau$-series employed rapidly diminish simple resummation procedures not addressing the Borel transformation may be applied. Use of Padé approximants $[\mathrm{L} / \mathrm{M}]$ looks quite reasonable in our case. First we perform the Padé resummation of the series for $\delta_{4}^{*}$ itself. Padé triangle for the pseudo- $\epsilon$ expansion (20) is presented in Table I. As one can see, Padé estimates converge to the value

$$
\delta_{4}^{*}=0.077 .
$$

An alternative estimate of the reduced anisotropy is obtained via Padé resummation of the $\tau$-series for $u^{*}$ and $v^{*}$. Corresponding Padé triangles are shown in Tables II and III. The values of coupling constants this resummation technique results in are seen to be:

$$
u^{*}=1.322, \quad v^{*}=0.182 .
$$

The first number almost coincides with its counterpart $u^{*}=1.321$ found by means of the conform-Borel analysis of the original six-loop expansions for $\beta$ functions $\frac{13}{\underline{ }}$ while the second one differs substantially from its $3 \mathrm{D}$ RG analog $v^{*}=0.117$. A substitution of the numbers (22) into (20) gives

$$
\delta_{4}^{*}=0.081,
$$

the value which is close to the Padé estimate (21). Averaging over these two numbers we obtain

$$
\delta_{4}^{*}=0.079 \pm 0.006
$$


This value may be referred to as a final estimate the pseudo- $\epsilon$ expansion machinery yields for the reduced anisotropy of cubic ferromagnet at criticality. In order to make this estimate realistic (certainly conservative) we accepted its inaccuracy to be three times bigger than the difference between Padé-approximant-based values (23) and (21).

\section{CRITICAL ANISOTROPY VERSUS $n_{c}$. WHAT ARE THE TRUE VALUES $\mathrm{OF} n_{c}$ AND $\delta_{4}^{*}$ ?}

So, the pseudo- $\epsilon$ expansion technique leads to the value of the reduced anisotropy which almost 1.5 times greater than its $\mathrm{RG}$ analogue $\delta_{4}^{*}=0.054$. How can one explain such a significant difference of the two field-theoretical estimates obtained within the highest-order available - six-loop - approximation? The roots of such a discrepancy may lie in some peculiarity of the $\tau$-series for $\delta_{4}^{*}$ : the numerical value of the anisotropy is much smaller than the coefficients of the first terms of the series (19), and it is calculated as a small difference of big numbers. The same is true, as seen from (18), for the coupling constant $v^{*}$.

This smallness, in its turn, reflects the fact that the boundary dimensionality of the order parameter $n_{c}$ is close to 3 . If $n_{c}$ coincided with the physical value of $n$, then the anisotropy parameter would be equal to zero at the critical point. Since the difference $3-n_{c}$ is numerically small $(0.1 \div 0.15)$, the values of $\delta_{4}^{*}$ and $v^{*}$ turn out to be small as well. However, precisely for this difference various field-theoretical schemes provide significantly different estimates. For example, the processing of the six-loop 3D RG expansions for $\beta$ functions of the cubic model by means of the conform-Borel technique leads to $3-n_{c}=0.11 \underline{13}$, while the values of this difference obtained by the resummation of the pseudo- $\epsilon$ and $\epsilon$ expansions for $n_{c}$ equal $0.138^{19}$ and $0.145^{\frac{17}{7}}$, respectively. Since the first of the above mentioned numbers differs from the others by tenths of percents, it is not surprising that a difference of values of $\delta_{4}^{*}$, obtained within the same iteration schemes, turns out to be significant.

To make the situation more transparent we calculate the cubic fixed point coordinate $v^{*}$, closely related to $\delta_{4}^{*}$, as a function of $n$. The curves $v^{*}(n)$ obtained by means of Padé resummation of the pseudo- $\epsilon$ expansion (11) under $n$ varying between 2.80 and 3.00 are shown in Fig. 1. Since diagonal Padé approximants are known to possess the best approximating properties, this figure contains curves given by the highest-order near-diagonal approximants $[3 / 2],[2 / 3]$, and by the diagonal one [2/2]. The value of $v^{*}$ the six-loop 3D RG analysis 
yields is also shown along with the values of marginal spin dimensionality $n_{c}$ extracted from the 6-loop 3D RG series $\frac{13}{2}$, from the $\epsilon$ expansion $\frac{13}{17}$, and from the pseudo- $\epsilon$ expansion $\frac{19}{1}$ for $n_{c}$. Comparing 3D RG and pseudo- $\epsilon$ expansions estimates one can clearly see that the closer $n_{c}$ is to 3 , the smaller $v^{*}$ and reduced anisotropy are at criticality.

Keeping in mind this point it is worthy to determine a real accuracy the pseudo- $\epsilon$ expansion technique provides when used to estimate the marginal spin dimensionality. Obviously, the accuracy of such an estimate is limited by the accuracy achieved in the course of evaluating the cubic fixed point coordinate and reduced anisotropy for $n$ under which $v^{*}(n)$ and $\delta^{*}(n)$ vanish. This accuracy, in its turn, may be characterized by the sensitivity of the numerical results with respect to iteration procedures employed. To get an idea about this sensitivity we calculate $n_{c}$ by means of solving equations

$$
v^{*}(n)=0, \quad \delta_{4}^{*}(n)=0
$$

with left-hand sides given by the series (11) and (16) respectively resummed using various Padé approximants. The results of these calculations are presented in Tables 4 and 5 .

Alternative estimates of $n_{c}$ thus obtained - 2.860 and 2.853 - are remarkably close to each other and to the value $n_{c}=2.862$ given by the Padé resummation of the pseudo- $\epsilon$ series for $n_{c}$ itself $\frac{19}{}$. Hence, the pseudo- $\epsilon$ expansion machinery may be thought of as a self-consistent iteration scheme. Moreover, the pseudo- $\epsilon$ expansion estimates agree well with the values 2.87 and 2.855 the resummation of the $\epsilon$ expansion for $n_{c}$ yields $\frac{13}{17}$. All this enables us to accept

$$
n_{c}=2.86 \pm 0.01
$$

as a most reliable numerical value of the marginal spin dimensionality. This number differs appreciably from the six-loop 3D RG estimate $n_{c}=2.89 \pm 0.04^{13}$ although both estimates are not in conflict since their uncertainty bars overlap. 3D RG estimates, however, look less accurate because of their sensitivity to the order of approximation. Indeed, 5-loop 3D RG estimate $n_{c}=2.91 \stackrel{12,13}{ }$ and its 6-loop analog differ from each other much stronger than their pseudo- $\epsilon$ expansion counterparts do. To demonstrate this we address the pseudo- $\epsilon$ series for $n_{c}$

$$
n_{c}=4-\frac{4}{3} \tau+0.2904231 \tau^{2}-0.1896725 \tau^{3}+0.1995126 \tau^{4}-0.224651 \tau^{5} .
$$

obtained from (11) by means of solving equation $v^{*}(n, \tau)=0$ iteratively. Numerical values of $n_{c}$ the Padé resummation of the series (27) yields are shown in Table 6. As is seen, the 
pseudo- $\epsilon$ expansion estimates rapidly converge to the asymptotic value and the deviation of the six-loop result from the five-loop one is really tiny (smaller than 0.0001) 43 .

With the value of $n_{c}$ in hand, we can get an alternative estimate of $v^{*}$. Since $v^{*}$ at $n=n_{c}$ is known to be zero and the difference $3-n_{c}=0.14$ is numerically small the value of $v^{*}$ for $n=3$ may be found from the power series

$$
v^{*}=\left.\frac{d v^{*}}{d n}\right|_{n_{c}}\left(3-n_{c}\right)+\left.\frac{1}{2} \frac{d^{2} v^{*}}{d n^{2}}\right|_{n_{c}}\left(3-n_{c}\right)^{2}+\ldots
$$

The derivatives entering (28) may be evaluated by means of the processing of their pseudo- $\epsilon$ expansions that are easily extracted from the series (11). Under $n=n_{c}=2.86$ they are as follows:

$$
\begin{gathered}
\left.\frac{d v^{*}}{d n}\right|_{n_{c}}=0.54580 \tau+0.46307 \tau^{2}+0.17409 \tau^{3}+0.07830 \tau^{4}-0.01880 \tau^{5}+0.01746 \tau^{6}, \\
\left.\frac{d^{2} v^{*}}{d n^{2}}\right|_{n_{c}}=-0.30398 \tau-0.31453 \tau^{2}-0.19609 \tau^{3}-0.17131 \tau^{4}-0.07026 \tau^{5}-0.09451 \tau^{6} .
\end{gathered}
$$

Good approximating properties of the $\tau$-series (29) are well pronounced, the expansion (30) also looks suitable for resummation. Use of Padé approximants to process these series results in

$$
\left.\frac{d v^{*}}{d n}\right|_{n_{c}}=1.25,\left.\quad \frac{d^{2} v^{*}}{d n^{2}}\right|_{n_{c}}=-1.26 .
$$

Taking into account that the higher-order contributions in (28) are negligibly small and substituting numbers (31) into this power series we get

$$
v^{*}=0.163, \quad \delta_{4}^{*}=0.0731 .
$$

The first number obtained differs from its counterpart (22) by $10 \%$ only while the second one is in agreement with the estimate (24). This allows us to adopt that the true values of universal quantities $n_{c}$ and $\delta_{4}^{*}$ lye within the uncertainty bars of our final estimates (24) and (26). This confirms also the conclusion that the pseudo- $\epsilon$ expansion machinery is the self-consistent procedure powerful enough to yield accurate numerical results.

So, use of the pseudo- $\epsilon$ expansion technique enabled us to refine the value of reduced anisotropy $\delta_{4}^{*}$ which turned out to be 1.5 times higher than that given by the conventional 3D RG analysis. This makes the arguments in favor of the possibility of experimental detection of the anisotropic critical behavior in cubic ferromagnets ${ }^{24}$ more convincing. 


\section{CONCLUSION}

To summarize, we have analyzed the behavior of nonlinear susceptibility of a cubic ferromagnet near Curie point within the pseudo- $\epsilon$ expansion approach. For the $n$-vector cubic model the six-loop pseudo- $\epsilon$ expansions for the cubic fixed point coordinates and reduced anisotropy have been derived for general $n$. Under the physical value $n=3$ these expansions have been found to possess a structure favorable for getting numerical estimates. Having processed the $\tau$-series for $u *$ and $v *$ and the pseudo- $\epsilon$ expansion for $\delta_{4}^{*}$ by means of the Padé approximants we've found the estimate $\delta_{4}^{*}=0.079 \pm 0.006$ which turned out to be considerably greater than its 3D RG counterpart. This discrepancy has been argued to reflect the fact that the value of the marginal spin dimensionality $n_{c}$ given by the six-loop 3D RG analysis differs appreciably from that obtained within the pseudo- $\epsilon$ expansion and $\epsilon$ expansion approaches. The evaluation of $v^{*}$ and $\delta_{4}^{*}$ via $n_{c}$ has been performed and has shown that alternative pseudo- $\epsilon$ expansion estimates are mutually consistent what allows to consider them as certainly reliable. The obtained value of $\delta_{4}^{*}$ is big enough to imply that the anisotropic critical behavior of cubic ferromagnets predicted by the theory is detectable in current physical and computer experiments.

\section{ACKNOWLEDGMENT}

We gratefully acknowledge the support of Saint Petersburg State University via Grant 11.38.636.2013 and of the Russian Foundation for Basic Research under Project 15-02-04687.

* Electronic address: ais2002@mail.ru

1 K. G. Wilson and M. E. Fisher, Phys. Rev. Lett. 28, 240 (1972).

2 D. J. Wallace, J. Phys. C, 6, 1390 (1973).

3 I. J. Ketley and D. J. Wallace, J. Phys. A, 6, 1667 (1973).

4 I. F. Lyuksyutov, V. L. Pokrovskii, Pis'ma v ZhETF, 21, 22 (1975) [JETP Lett. 21, 9 (1975)].

5 A. Aharony, Phys. Rev. B 8, 4270 (1973).

6 A. I. Sokolov, Fiz. Tverd. Tela 19, 747 (1977) [Sov. Phys. Solid State 19, 433 (1977)].

7 K. E. Newman and E. K. Riedel, Phys. Rev. B 25, 264 (1982). 
8 M. Ferer, J. P. Van Dyke, and W. J. Camp, Phys. Rev. B 23, 2367 (1981).

9 I. O. Maier, A. I. Sokolov, Izvestiya AN SSSR, seriya fizicheskaya 51, 2103 (1987); I. O. Maier and A. I. Sokolov, Ferroelectrics Lett. 9, 95 (1988).

10 N. A. Shpot, Phys. Lett. A 142, 474 (1989).

11 I. O. Mayer, A. I. Sokolov, and B. N. Shalaev, Ferroelectrics 95, 93 (1989);

12 D. V. Pakhnin and A. I. Sokolov, Phys. Rev. B 61, 15130 (2000).

13 J. M. Carmona, A. Pelissetto, and E. Vicari, Phys. Rev. B 61, 15136 (2000).

14 H. Kleinert and V. Schulte-Frohlinde, Phys. Lett. B 342, 284 (1995).

15 H. Kleinert and S. Thoms, Phys. Rev. D 52, 5926 (1995).

16 H. Kleinert, S. Thoms, and V. Schulte-Frohlinde, Phys. Rev. B 56, 14428 (1997).

17 B. N. Shalaev, S. A. Antonenko, and A. I. Sokolov, Phys. Lett. A 230105 (1997).

18 R. Folk, Yu. Holovatch, and T. Yavors'kii, Phys. Rev. B 61, 15114 (2000).

19 R. Folk, Yu. Holovatch, and T. Yavors'kii, Phys. Rev. B 62, 12195 (2000).

20 K. B. Varnashev, Phys. Rev. B 61, 14660 (2000).

21 A. Pelissetto and E. Vicari, Phys. Reports 368, 549 (2002).

22 M. Hasenbusch and E. Vicari, Phys. Rev. B 84, 125136 (2011).

23 R. Guida and J. Zinn-Justin, J. Phys. A 31, 8103 (1998).

24 D. V. Pakhnin and A. I. Sokolov, Phys. Rev. B 64, 094407 (2001).

25 J. C. Le Guillou and J. Zinn-Justin, Phys. Rev. B 21, 3976 (1980).

26 C. von Ferber, Yu. Holovatch, Phys. Rev. E 56, 6370 (1997).

27 C. von Ferber, Yu. Holovatch, Phys. Rev. E 59, 6914 (1999).

28 Yu. Holovatch, M. Dudka, and T. Yavorskii, J. Phys. Stud. 5, 233 (2001).

29 M. Dudka, Yu. Holovatch, and T. Yavorskii, Acta Phys. Slov. 52, 323 (2002).

30 P. Calabrese and P. Parruccini, J. High Energy Phys. 05, 018 (2004).

31 Yu. Holovatch, D. Ivaneiko, and B. Delamotte, J. Phys. A 37, 3569 (2004).

32 M. Dudka, Yu. Holovatch, and T. Yavorskii, J. Phys. A 37, 10727 (2004).

33 A. I. Sokolov and M. A. Nikitina, Phys. Rev. E 89, 052127 (2014).

34 A. I. Sokolov and M. A. Nikitina, Phys. Rev. E 90, 012102 (2014).

35 M. A. Nikitina and A. I. Sokolov, Teor. Mat. Fiz. 186, 230 (2016) [Theor. Math. Phys. 186, 192 (2016)].

36 A. I. Sokolov and M. A. Nikitina, Physica A 444, 177 (2016). 
37 P. Calabrese, E. V. Orlov, D. V. Pakhnin, and A. I. Sokolov, Phys. Rev. B 70, 094425 (2004).

38 P. Calabrese and P. Parruccini, Phys. Rev. B 71, 064416 (2005).

39 A. I. Sokolov, Fiz. Tverd. Tela 47, 2056 (2005) [Phys. Sol. State 47, 2144 (2005)].

40 A. I. Sokolov, Teor. Mat. Fiz. 176, 140 (2013) [Theor. Math. Phys. 176, 948 (2013)].

41 M. A. Nikitina and A. I. Sokolov, Phys. Rev. E 89, 042146 (2014).

42 A. L. Korzhenevskii, Zh. Eksp. Teor. Fiz. 71, 1434 (1976) [Sov. Phys. JETP 44, 751 (1976)].

43 The series (27) and numbers in Table 6 are in a very good aggrement with those obtained in Ref. [19]. In this paper, the pseudo- $\epsilon$ expansion for $n_{c}$ was resummed by means of the Padé-BorelLeroy technique and high stability of the numerical results with respect to the resummation procedure was demonstrated. 
TABLE I: Padé triangle for the pseudo- $\epsilon$ expansion (20) of the reduced anisotropy $\delta_{4}$ in the Curie point. Approximant [2/1] has a pole close to 1 ("dangerous"), corresponding estimate is not reliable and therefore is given in brackets. The bottom line $(\mathrm{RoC})$ shows the rate and the character of convergence of Padé estimates to the asymptotic value. Here Padé estimate of $k$-th order is the number given by corresponding diagonal approximant $[\mathrm{L} / \mathrm{L}]$ or by a half of the sum of the values given by approximants $[\mathrm{L} / \mathrm{L}-1]$ and $[\mathrm{L}-1 / \mathrm{L}]$ when a diagonal approximant is absent.

\begin{tabular}{c|cccccc}
$M \backslash L$ & 0 & 1 & 2 & 3 & 4 & 5 \\
\hline 0 & -0.33333 & -0.03704 & 0.02960 & 0.08257 & 0.05734 & 0.09583 \\
1 & -0.17647 & 0.04893 & $(0.28797)$ & 0.06548 & 0.07258 & \\
2 & -0.11578 & 0.13681 & 0.07997 & 0.07700 & & \\
3 & -0.08139 & 0.03850 & 0.07688 & & & \\
4 & -0.06127 & 0.17108 & & & & \\
5 & -0.04705 & & & & & \\
\hline RoC & -0.33333 & -0.10675 & 0.04893 & $(0.21239)$ & 0.07997 & 0.07694
\end{tabular}

TABLE II: Padé table for the pseudo- $\epsilon$ expansion (18) of the coupling constant $u^{*}$. Approximants are constructed for $u^{*} / \tau$, i. e. neglecting the insufficient factor $\tau$. Approximants [3/1], [3/2] and $[2 / 2]$ have dangerous poles, therefore corresponding estimates are not reliable; in the table they are bracketed. The bottom line (RoC) indicates the character of convergence of Padé estimates to the asymptotic value. Here the Padé estimate of $k$-th order is the number obtained in the same manner as in the case of $\delta_{4}^{*}$ (Table I). The final value $u^{*}=1.3218$ is the result of averaging over three working approximants $[4 / 1],[2 / 3]$, and [1/4].

\begin{tabular}{c|cccccc}
$M \backslash L$ & 0 & 1 & 2 & 3 & 4 & 5 \\
\hline 0 & 1.2222 & 1.5546 & 1.4320 & 1.3664 & 1.3053 & 1.3180 \\
1 & 1.6787 & 1.4650 & 1.2909 & $(0.4782)$ & 1.3158 & \\
2 & 1.3545 & 1.3832 & $(0.9025)$ & $(1.2483)$ & & \\
3 & 1.3868 & 1.3629 & 1.3197 & & & \\
4 & 1.3004 & 1.3300 & & & & \\
5 & 1.3472 & & & & & \\
\hline RoC & 1.2222 & 1.6166 & 1.4650 & 1.3371 & $(0.9025)$ & 1.3218
\end{tabular}


TABLE III: Padé triangle for pseudo- $\epsilon$ expansion (19) of the coupling constant $v^{*}$. Approximants are constructed for $v^{*} / \tau$, i. e. with the factor $\tau$ omitted. Approximant [1/1] has a dangerous pole, corresponding estimate is unreliable and presented in brackets. The convergence of Padé estimates to the asymptotic value is illustrated by the bottom line (RoC). Here the Padé estimate of $k$-th order is the same as in Table 1.

\begin{tabular}{c|cccccc}
$M \backslash L$ & 0 & 1 & 2 & 3 & 4 & 5 \\
\hline 0 & -0.4074 & -0.2768 & -0.0444 & 0.0840 & 0.1342 & 0.1565 \\
1 & -0.3085 & $(-0.5753)$ & 0.2426 & 0.1665 & 0.1741 & \\
2 & -0.2043 & 0.0416 & 0.1507 & 0.1729 & & \\
3 & -0.1505 & 0.1905 & 0.1906 & & & \\
4 & -0.1149 & 0.1906 & & & & \\
5 & -0.0900 & & & & & \\
\hline RoC & -0.4074 & -0.2926 & $(-0.5753)$ & 0.1416 & 0.1507 & 0.1817
\end{tabular}

TABLE IV: Values of $n_{c}$ calculated by solving the equation $v^{*}(n)=0$ with the left-hand side equal to Padé-resummed pseudo- $\epsilon$ expansion (11). Padé approximants $[\mathrm{L} / \mathrm{M}]$ are constructed for $v^{*} / \tau$, i. e. neglecting the trivial factor $\tau$. Approximants $[1 / 1]$ and $[1 / 3]$ have poles very close to the knots of interest, corresponding estimates are not reliable and absent in the table. The bottom line (RoC) indicates the character of convergence of estimates to the asymptotic value. Here the estimate of $k$-th order is the number obtained in the same manner as in Table I.

\begin{tabular}{c|cccccc}
$M \backslash L$ & 0 & 1 & 2 & 3 & 4 & 5 \\
\hline 0 & 4.0000 & 3.3268 & 3.0419 & 2.9278 & 2.8842 & 2.8673 \\
1 & 4.0000 & - & 2.8056 & 2.8479 & 2.8571 & \\
2 & 4.0000 & 2.9651 & 2.8542 & 2.8601 & & \\
3 & 4.0000 & - & 2.8594 & & & \\
4 & 4.0000 & 2.8723 & & & & \\
5 & 4.0000 & & & & & \\
\hline RoC & 4.0000 & 3.6634 & - & 2.8854 & 2.8542 & 2.8598
\end{tabular}


TABLE V: Values of $n_{c}$ extracted from the equation $\delta^{*}(n)=0$ with the left-hand side given by $\tau$ series (16) resummed using Padé approximants [L/M]. Two estimates are absent since approximant $[2 / 1]$ is spoiled with dangerous pole while approximant [1/4] has no real knots. The bottom line (RoC) indicates the character of convergence of estimates to the asymptotic value where the estimate of $k$-th order is the number obtained in the same way as in Table I.

\begin{tabular}{c|cccccc}
$M \backslash L$ & 0 & 1 & 2 & 3 & 4 & 5 \\
\hline 0 & 4.0000 & 3.0704 & 2.9457 & 2.8517 & 2.8899 & 2.8217 \\
1 & 4.0000 & 2.9092 & - & 2.8784 & 2.8649 & \\
2 & 4.0000 & 2.7255 & 2.8501 & 2.8528 & & \\
3 & 4.0000 & 2.9244 & 2.8528 & & & \\
4 & 4.0000 & - & & & & \\
5 & 4.0000 & & & & & \\
\hline $\mathrm{RoC}$ & 4.0000 & 3.5352 & 2.9092 & - & 2.8501 & 2.8528
\end{tabular}

TABLE VI: Padé triangle for pseudo- $\epsilon$ expansion (27) of the marginal spin dimensionality $n_{c}$. Approximant $[1 / 2]$ has a pole very close to 1 , corresponding estimate being unreliable is bracketed. The convergence of Padé estimates to the asymptotic value is illustrated by the bottom line (RoC) where the Padé estimate of $k$-th order is the number obtained in the same way as in Table 1 .

\begin{tabular}{c|cccccc}
$M \backslash L$ & 0 & 1 & 2 & 3 & 4 & 5 \\
\hline 0 & 4.00000 & 2.66667 & 2.95709 & 2.76742 & 2.96693 & 2.74228 \\
1 & 3.00000 & 2.90515 & 2.84235 & 2.86456 & 2.86126 & \\
2 & 2.91579 & $(2.06451)$ & 2.86156 & 2.86162 & & \\
3 & 2.84113 & 2.87113 & 2.86162 & & & \\
4 & 2.89218 & 2.86384 & & & & \\
5 & 2.82985 & & & & & \\
\hline RoC & 4.00000 & 2.83333 & 2.90915 & - & 2.86156 & 2.86162
\end{tabular}




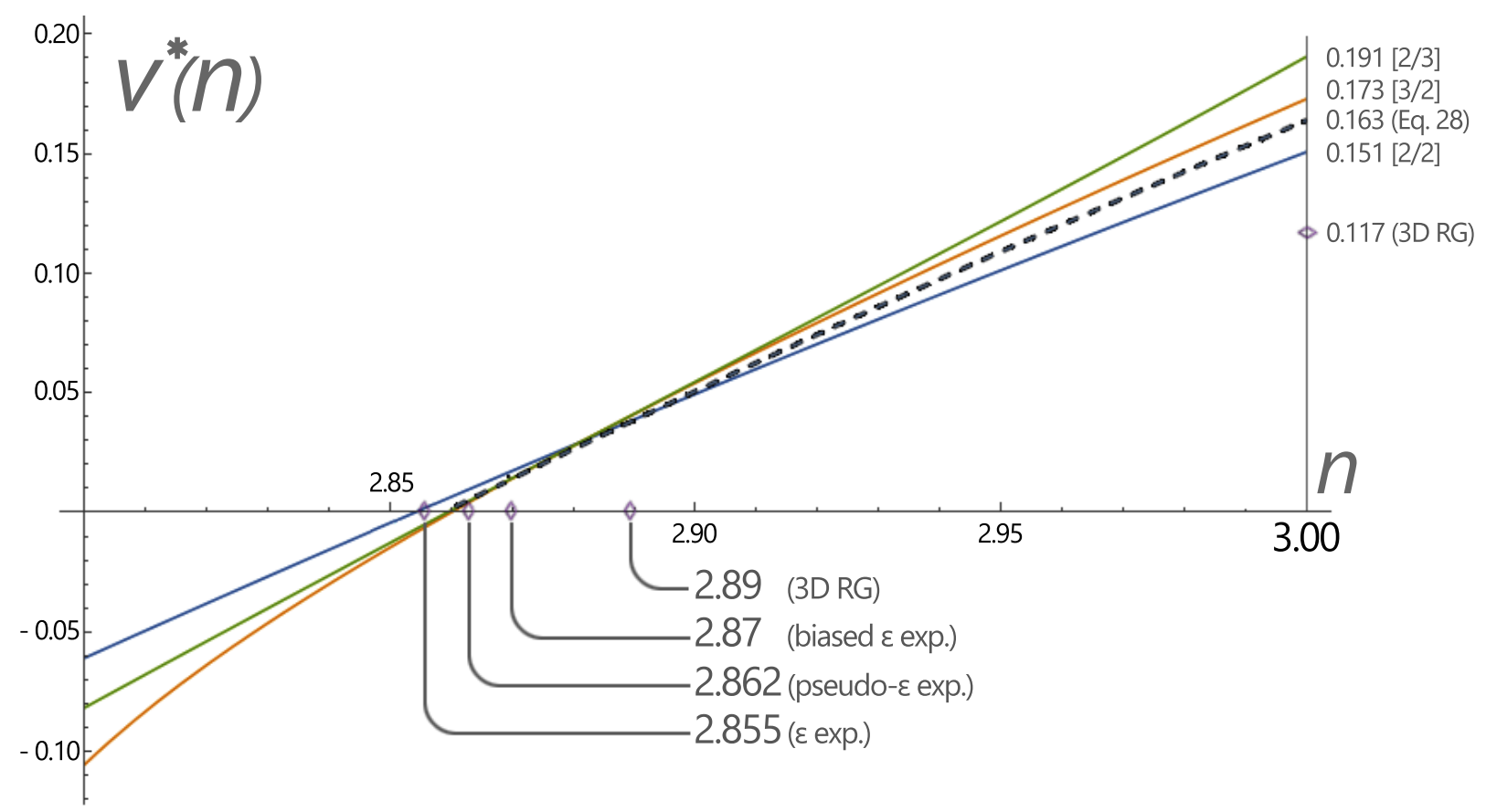

FIG. 1: (Color online) The cubic fixed point coordinate $v^{*}$ as a function of $n$ obtained by means of Padé resummation of $\tau$-series (11). Three curves given by the diagonal [2/2] and near-diagonal approximants [3/2], [2/3] for $v^{*} / \tau$, i. e. with insignificant factor $\tau$ omitted, are shown. The values of marginal spin dimensionality $n_{c}$ found by the analysis of 6-loop 3D RG series $\frac{13}{3}(2.89)$ and extracted from the $\epsilon$ expansion $\underline{13}, \underline{17}(2.87,2.855)$ and from the pseudo- $\epsilon$ expansion $\underline{19}$ (2.862) for $n_{c}$ are marked with diamonds. The diamond on the right vertical axis shows the value of $v^{*}$ the six-loop 3D RG analysis yields. The dashed line is the curve $v^{*}(n)$ given by the powers series in $\left(n-n_{c}\right)$ which arrives to 0.163 when the spin dimensionality approaches the physical value $n=3$. 